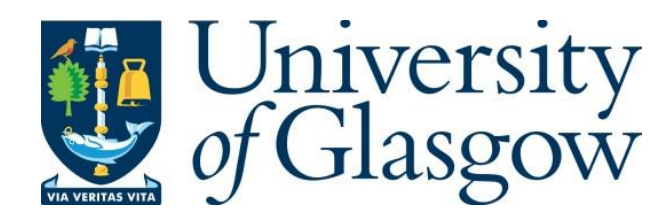

Almeida, T. P., McGrouther, D., Kovács, A., Dunin-Borkowski, R. E. and McVitie, S. (2020) Effect of annealing on the magnetic states of FEBID-grown cobalt nanopatterns examined by off-axis electron holography. Journal of Microscopy, 279(3), pp. 217-221.

This is the peer reviewed version of the following article, Almeida, T. P., McGrouther, D., Kovács, A., Dunin-Borkowski, R. E. and McVitie, S. (2020) Effect of annealing on the magnetic states of FEBID-grown cobalt nanopatterns examined by off-axis electron holography. Journal of Microscopy, 279(3), pp. 217-221, which has been published in final form at http://dx.doi.org/10.1111/jmi.12869

This article may be used for non-commercial purposes in accordance with Wiley Terms and Conditions for Self-Archiving.

http://eprints.gla.ac.uk/208510/

Deposited on: 27 January 2020

Enlighten - Research publications by members of the University of Glasgow http://eprints.gla.ac.uk 


\title{
Effect of annealing on the magnetic states of FEBID-grown cobalt nanopatterns examined by off-axis electron holography
}

\author{
Trevor P. Almeida ${ }^{1 *}$, Damien McGrouther ${ }^{1}$, András Kovács ${ }^{2}$, Rafal E. Dunin-Borkowski² \\ and Stephen McVitie ${ }^{1}$
}

\author{
${ }^{1}$ SUPA, School of Physics and Astronomy, University of Glasgow, Glasgow, G12 8QQ, UK. \\ ${ }^{2}$ Ernst Ruska-Centre for Microscopy and Spectroscopy with Electrons (ER-C) and Peter Grünberg \\ Institute, Forschungszentrum Jülich, D-52425 Jülich, Germany.
}

\begin{abstract}
The growth of cobalt nanopatterns (NPs) using focused electron-beam induced deposition (FEBID) for localised magnetic studies is presented. The initial FEBID products are shown to be polycrystalline and form hetero-structured core-shell NPs through surface oxidation. Off-axis electron holography is performed to reconstruct their morphology, thickness profile and image their individual magnetic vortex domain states. In-situ annealing to $400^{\circ} \mathrm{C}$ promoted migration of the Co-overspray to grow the Co NPs and improved their crystallinity through coarsening, as well as induced diffusion of embedded carbon out of their surface. It is found that the change in their morphology and chemical instability under heating restricts their suitability for examining thermally induced magnetic variations.
\end{abstract}

\section{Introduction}

The demand for improved functionality in magnetoelectronic devices is resulting in the development of innovative combinations of hard and soft magnetic materials, which are referred to as exchange-spring magnets. In a bi-magnetic core-shell (CS) nanostructure, exchange coupling across the CS interface facilitates cooperative magnetic switching and provides tuneable magnetic properties $^{1,2}$. However, our current understanding of the CS exchange interaction is limited to bulk magnetic measurements and micromagnetic modelling, driving the need for more localised studies using transmission electron microscopy (TEM) techniques. CS nanostructures are commonly synthesized as powders or dispersions through chemical routes including co-precipitation, hydrothermal synthesis, etc., but often exhibit a wide size distribution, agglomerate easily and are difficult to isolate for localized magnetic studies without inter-particle interactions ${ }^{3}$. Modern scanning electron microscopes (SEMs) can incorporate systems for the injection of elementcontaining gases that can be used as precursors for creating magnetic nanopatterns (NPs), with feature sizes as small as $10 \mathrm{~nm}$, using focused electron beam induced deposition (FEBID) $)^{4-6}$. In FEBID, the electron beam is used to dissociate the precursor gas near a substrate and locally deposit the carried metal in the same shape as the scanned beam. Alteration of the electron-beam scan conditions during beam-induced decomposition of the injected gases provides effective control over the size, morphology and inter-spacing of the deposited magnetic material. Further, the deposition of reactive ferromagnetic metals such as $\mathrm{Co}^{7-9}$ can result in the formation of CS NPs through surface oxidation. Hence, we are in a timely position to synthesise isolated CS systems using FEBID and use the TEM technique of off-axis electron holography to investigate the effect of localized magnetic exchange coupling between their metal-core and oxide-shell. One useful method to induce dynamic magnetic processes that provide access to the effect of exchange coupled CS systems is through in-situ heating. However, to isolate the effects on magnetism we must first understand the effect of heating on the original FEBID-grown CS NPs. In the present study, we investigate the effect of annealing on structural, chemical and magnetic properties of CS NPs using a series of advanced in-situ TEM techniques. 


\section{Experimental}

FEBID is used to synthesise a range of Co NPs on electron-transparent SiN membranes of DENSSolutions Wildfire $^{\mathrm{TM}}$ TEM electronic (e-) chips, with two Co NPs presented here $(\sim 90 \mathrm{~nm}$ in diameter). $\mathrm{Co}_{2}(\mathrm{CO})_{8}$ was used as a precursor for the elemental deposition of $\mathrm{Co}$, with the gas injection system (GIS) needle inserted to the standard height relative to the $\mathrm{SiN}$ membrane located at the working distance of $3.9 \mathrm{~mm}$ from the SEM column pole piece. The GIS needle has a diameter of $300 \mu \mathrm{m}$ and was positioned $70 \mu \mathrm{m}$ from the SEM beam, at an angle of $48^{\circ}$ relative to the optic axis. The precursor crucible was heated to $28^{\circ} \mathrm{C}$ prior to the start of the gas flow and the growth was controlled through systematic variation of the SEM electron beam voltages $(5 \mathrm{kV}-15 \mathrm{kV})$ and beam currents (0.34nA-0.69nA). Conventional and scanning TEM imaging of the FEBID-grown NPs was performed using a Thermo Fisher Titan G2 80-200 or Tecnai G2 F20 (both ER-C) at $200 \mathrm{kV}$, with structural analysis provided by selected area electron diffraction (SAED). Energy dispersive X-ray (EDX) spectroscopy allowed for chemical mapping of the elemental distribution across the CS NPs. Off-axis electron holograms were acquired at $300 \mathrm{kV}$ in Lorentz mode in a Titan 80-300 TEM (ER-C) equipped with an image aberration corrector, rotatable electron biprisms and recorded using a Gatan K2 high-speed direct electron detection camera. The total phase shift of the electron wave recorded in one dimension, $\phi(x)$, is sensitive to the electrostatic potential, $\phi_{e}$, and the in-plane component of the magnetic induction, $\phi_{m}$, in the specimen, as summarised by:

$$
\phi(x)=\phi_{e}+\phi_{m}=C_{E} \int V(x, z) d z-\left(\frac{2 \pi e}{h}\right) \int A_{z}(x, z) d z
$$

where the incident electron beam direction $z$ is perpendicular to $x, C_{E}$ is an interaction constant that takes a value of $6.53 \times 10^{6} \mathrm{radV}^{-1} \mathrm{~m}^{-1}$ at an accelerating voltage of $300 \mathrm{kV}, V$ is the electrostatic potential and $A_{z}$ is the component of the magnetic vector potential along $z$. Absent from additional charge redistribution around the sample, $V$ comprises primarily the mean inner potential (MIP) of the material $V_{0}$. If the MIP of material does not vary within its thickness $t$, then $\phi_{e}$ can be simplified to $\phi_{e}=C_{E} V_{o t}$. Each NP was initially magnetized in opposite directions by tilting the sample by $\pm 75^{\circ}$ and turning on the conventional microscope objective lens to apply a saturating magnetic field of $>1.5 \mathrm{~T}$. This allowed for the cancellation of the net magnetic contribution to the phase in order to determine the MIP contribution, and through subtraction of this MIP contribution the in-plane magnetic induction in one of the two tilt directions was isolated. For the purpose of examining the effect of annealing, holograms were acquired in magnetic-fieldfree conditions at $25{ }^{\circ} \mathrm{C}$ before and after in situ heating to $400{ }^{\circ} \mathrm{C}$ for 10 minutes under vacuum using the DENSSolutions Wildfire ${ }^{\mathrm{TM}}$ heating holder, as described previously ${ }^{10,11}$. For construction of the magnetic induction maps, the cosine of the magnetic contribution was amplified to produce phase contours and colours were added to show the direction of the projected induction (denoted by the colour wheels). The outlines of the NPs were determined using an appropriate threshold on the MIP contribution to the phase shift. 

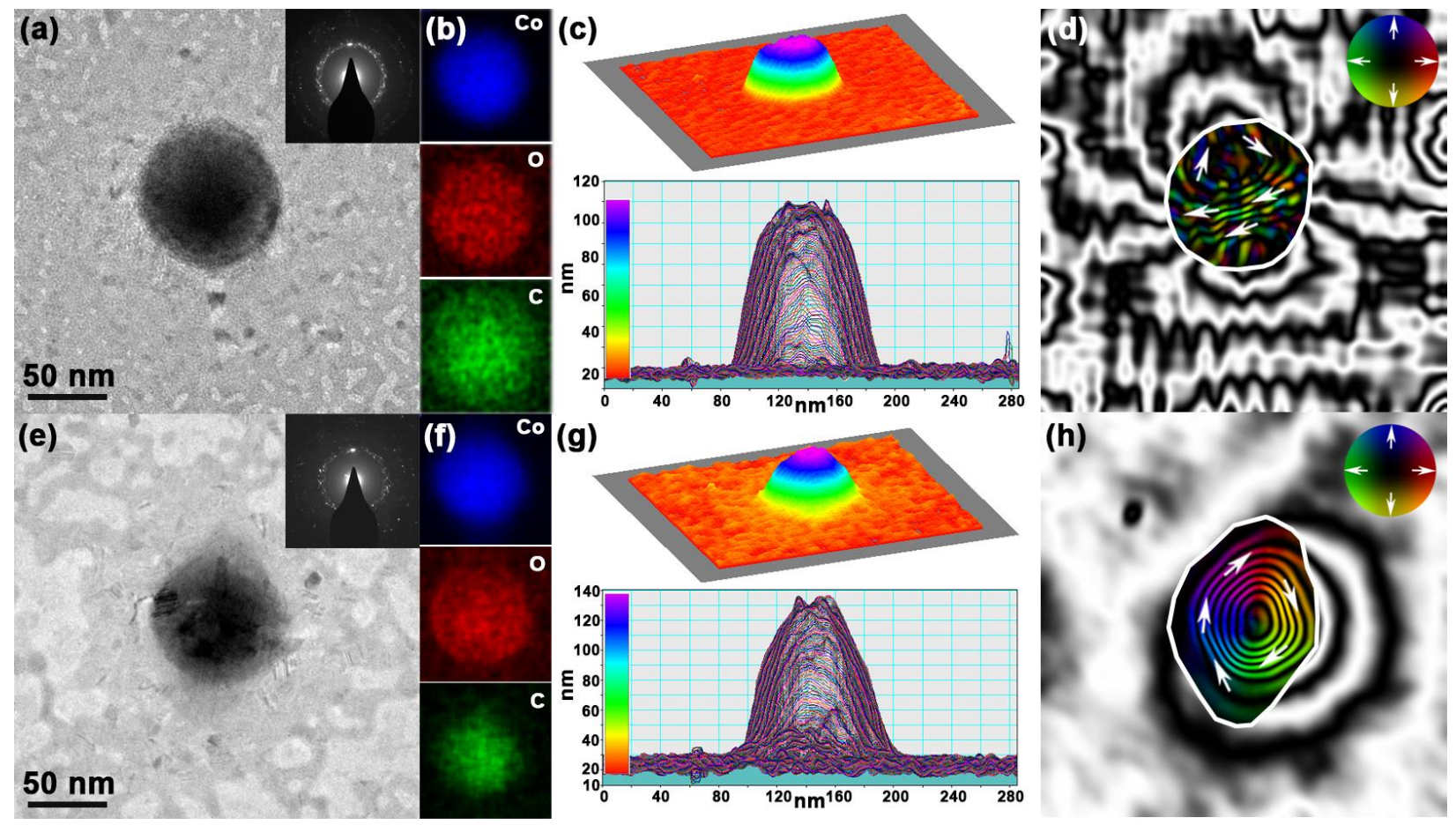

Figure 1. (a,e) BF TEM images of a Co NP (a) before; and (e) after in-situ heating to $400^{\circ} \mathrm{C}$, with SAED patterns (inset). (b,f) Associated EDX chemical maps showing the elemental distribution of cobalt, oxygen and carbon. (c,g) Morphology and thickness profile of the Co NP estimated from the $\phi_{e}$ and constant MIP. The colour in the top panel represents height and is defined by the scale bar in the thickness profile (bottom panel, inset). (d,h) Magnetic induction maps reconstructed from holograms acquired (d) before; and (h) after in-situ heating to $400^{\circ} \mathrm{C}$. The contour spacing is (d) 0.049; and (h) 0.785 radians and magnetisation direction is shown using arrows, as depicted in the colour wheel.

\section{Results}

Figure 1 presents the effect of annealing on a FEBID-grown Co NP, providing information on the changes of its size, chemical composition, localised structure, morphology and magnetisation. The bright field (BF) TEM image of Fig. 1a provides a localised view of the native Co NP ( $90 \mathrm{~nm}$ in diameter) at $25^{\circ} \mathrm{C}$ with obvious regions of Co overspray from FEBID process in its close vicinity, whilst the diffraction rings in the corresponding SAED pattern (Fig. 1c) reveals the Co NP to be polycrystalline. The EDX chemical maps (Fig. 1b) show the Co distribution to be uniform across the NP but exhibits an O-rich surface layer, confirming the formation of an Co / Co-oxide CS structure. A large $\mathrm{C}$ signal is also detected and is considered due to embedding of residual hydrocarbon molecules remaining as a reaction product of the dissociated $\mathrm{Co}_{2}(\mathrm{CO})_{8}$ precursor ${ }^{12}$. Fig. 1c presents the morphology and thickness profile ( $110 \mathrm{~nm}$ maxima) of the Co NP, determined using the equation $\phi_{e}=C_{E} V_{0} t$, where $V_{0}=25.0 \mathrm{~V}$ is estimated by averaging several values of MIP for $\mathrm{Co}^{13-15}$. Fig. 1d displays a magnetic induction map of the Co NP and the central contours flowing from right to left could be misinterpreted as a uniformly magnetized state. However, due to its size and part of the flux loops either side of the central magnetic contours being contained within the Co NP, it is more likely to be a vortex state that is observed edgeon ${ }^{16,17}$. Fig. 1e demonstrates the effect of in-situ annealing to $400^{\circ} \mathrm{C}$, revealing migration and build-up of the Co overspray on the Co NP. The EDX maps of Fig. If display a pronounced O signal at the Co NP edge and depletion of $50 \%$ of total atomic C\% from the NP surface, as shown in the summary of atomic \% before and after annealing (Table 1.). The associated morphology and thickness profile (Fig. 1g) reveal amplified overspray roughness and an increase in height of the Co NP by $\sim 25 \mathrm{~nm}$. The magnetic induction map of Fig. 1h clearly shows a change to a vortex state flowing in a clockwise direction with a stray magnetic field. 

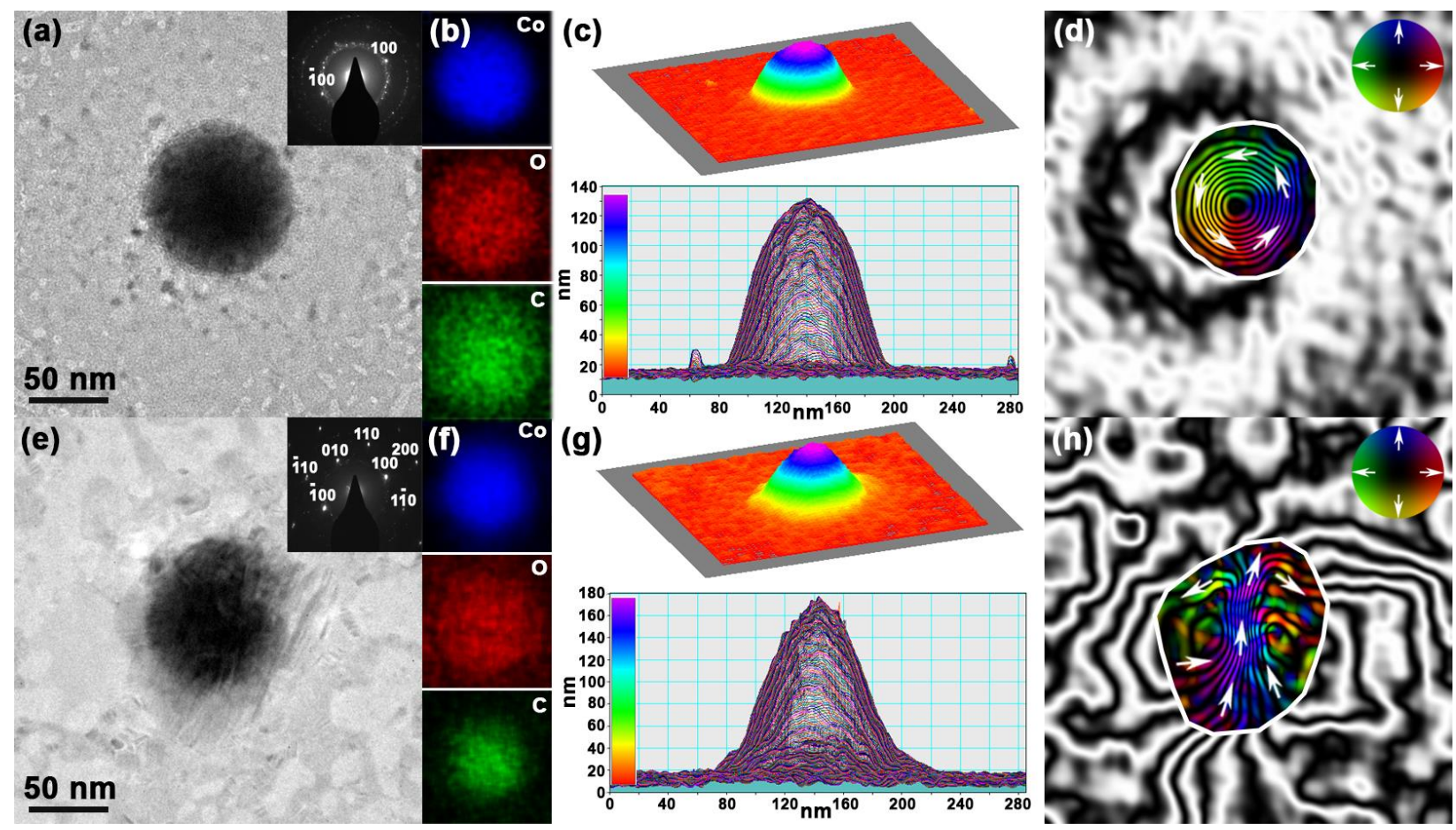

Figure 2. (a,e) BF TEM images of a Co NP (a) before; and (e) after in-situ heating to $400^{\circ} \mathrm{C}$, with SAED patterns (inset). (b,f) Associated EDX chemical maps showing the elemental distribution of cobalt, oxygen and carbon. (c,g) Morphology and thickness profile of the Co NP estimated from the $\phi_{e}$ and constant MIP. The colour in the top panel represents height and is defined by the scale bar in the thickness profile (bottom panel, inset). (d,h) Magnetic induction maps reconstructed from holograms acquired (d) before; and (h) after in-situ heating to $400^{\circ} \mathrm{C}$. The contour spacing is (d) 0.524; and (h) 0.131 radians and magnetisation direction is shown using arrows, as depicted in the colour wheel.

Similar to Figure 1, Figure 2 presents the effect of annealing on the second FEBID-grown Co NP. The BF TEM image (Fig. 2a) shows a similar Co NP ( $\sim 90 \mathrm{~nm}$ in diameter) and Co overspray, with bright spots in the SAED pattern corresponding to the $\{100\}$ set of planes of $\mathrm{Co}_{3} \mathrm{O}_{4}$. The EDX chemical maps (Fig. 2b) show similar elemental distributions of $\mathrm{Co}, \mathrm{O}$ and $\mathrm{C}$ as the Co NP in Fig. 1b, and Fig. 2c reveals it to be analogous in morphology but slightly thicker at $\sim 130 \mathrm{~nm}$. The magnetic induction map of Fig. $2 \mathrm{~d}$ clearly shows a vortex state flowing in an anti-clockwise direction. Annealing of the Co NP at $400^{\circ} \mathrm{C}$ again results in migration and build-up of the Co overspray (Fig. 2e), along with an increase in intensity of the diffraction spots in SAED pattern (inset), indexed to $\mathrm{Co}_{3} \mathrm{O}_{4}$. The EDX maps of Fig. $2 \mathrm{f}$ display do not reveal much change in the $\mathrm{Co}$ NP apart from depletion of 50\% of total atomic C\% from the NP surface (Table 1), whilst Fig. $2 \mathrm{~g}$ reveals amplified overspray roughness, an increase in height of the Co NP by $\sim 30 \mathrm{~nm}$ and widening of its base. The magnetic induction map of Fig. $2 \mathrm{~h}$ shows magnetic contours flowing from bottom to top through the Co NP with flux closure either side, indicative of a vortex core aligned along the vertical axis with a stray magnetic field.

\begin{tabular}{|l|c|c|c|c|}
\hline & \multicolumn{2}{|c|}{ Co NP in Fig. 1 } & \multicolumn{2}{c|}{ Co NP in Fig. 2 } \\
\hline Element & Pre-anneal atom\% & Post-anneal atom\% & Pre-anneal atom\% & Post-anneal atom\% \\
\hline Carbon & 51.9 & 26.4 & 50.1 & 25.4 \\
\hline Oxygen & 24.5 & 30.8 & 23.9 & 28.0 \\
\hline Cobalt & 13.1 & 25.8 & 16.6 & 29.0 \\
\hline Nitrogen & 8.5 & 13.2 & 7.6 & 14.5 \\
\hline Silicon & 2.0 & 3.6 & 1.8 & 3.1 \\
\hline
\end{tabular}

Table 1. Quantification from the entire EDX chemical maps in Figs $1 \& 2$, before and after annealing. 


\section{Discussion}

This TEM investigation has provided chemical, magnetic and structural characterisation of two Co NPs grown by FEBID and demonstrated the effect of $i n$-situ annealing to $400^{\circ} \mathrm{C}$. It is clear the FEBID technique has successfully deposited two circular Co NPs $\sim 90 \mathrm{~nm}$ in diameter on the SiN windows of the Wildfire e-chips, along with Co overspray. The as-grown Co NPs are found to be polycrystalline and EDX analysis reveals they include a significant amount of carbon. This is consistent with previous studies showing the deposits grown at similarly low electron beam current comprise small isolated cobalt nanocrystals ( 5 to $7 \mathrm{~nm}$ in size) embedded in an amorphous carbon matrix $^{18}$. EDX analysis also confirms the formation of an oxide-rich shell around the highlyreactive Co NPs, considered due to exposure to ambient conditions after the FEBID process. Using the relationship $\phi_{e}=C_{E} V_{0} t$ provides a useful method to visualise the morphology and thickness profile of the Co NPs, assuming they are grown with a flat base of the SiN membrane. This would perhaps not be appropriate for drop-cast nanoparticle powders. Whilst the two native Co NPs exhibit similar dimensions, the magnetic induction maps both reveal vortex states but at different orientations.

The in-situ annealing to $400^{\circ} \mathrm{C}$ has had a significant effect on both Co NPs, most notably the increased roughness of the Co overspray and its apparent migration into the Co NPs. This has resulted in widening of their base, particularly in Fig. $2 \mathrm{~g}$, and an increase in their height by $\sim 25-$ $30 \mathrm{~nm}$, assuming their $V_{0}$ does not change significantly with annealing. Another pronounced effect is concentration of the carbon signal in the EDX maps towards the centre of the Co NPs. In-situ annealing has been shown to increase the relative Co content in FEBID grown nanostructures through coarsening growth of crystallites ${ }^{19}$ and is considered to induce C-diffusion from the Co NP surface, resulting in a 50\% reduction in atomic C \% for both NPs. This coarsening effect is confirmed by the SAED patterns, with Fig. 2e (inset) suggesting the in-situ annealing has promoted a single crystalline $\mathrm{Co}_{3} \mathrm{O}_{4}$ shell. The annealing also had a marked effect on the magnetic properties of the Co NPs, and it is considered the variation in remanent domain states are due to the morphology-dependent changes in shape anisotropy. The main difference between the magnetic induction maps in figure $1 \& 2$ is that with annealing, the vortex states change from side-on view to a view a down the vortex core (Figure 1), and vice versa (Figure 2). Whilst annealing is known to increase the net magnetization strength in FEBID-grown Co nanostructures ${ }^{19}$, these changes in orientation of the magnetic states prevent direct comparison of quantitative measurements of inplane induction. This is because electron holography is only sensitive to the in-plane component of the magnetic induction. Hence these differently oriented magnetic states could present a large proportion of magnetic induction either in-plane or out-of-plane by comparison, which would explain the difference in phase contours in the magnetic induction maps. The marked chemical and structural changes induced by annealing at $400^{\circ} \mathrm{C}$ imply the FEBID-grown NPs are not ideal candidates for investigating temperature induced changes in vortex domain states, which are known to be thermally stable up to relatively high temperatures ${ }^{20}$. However, this study has demonstrated that in-situ annealing can be used to improve the crystallinity and, with improved control, tune the net magnetization of individual NPs or large complex arrays produced by FEBID, for potential use in magnetic data storage.

In summary, FEBID has successfully grown isolated CS Co NPs $\sim 90 \mathrm{~nm}$ on in-situ TEM chips. Off-axis electron holography reveals their morphology, thickness and vortex magnetic domain states. In-situ annealing induced migration of the Co-overspray to grow the Co NPs and increase their crystallinity, but their instability under heating restricts their suitability for examining thermally induced magnetic processes. 


\section{References}

[1] G. C. Lavorato, et al., J. Alloys Compd. 633, 333-337 (2015).

[2] J-H. Lee et al., Nat. Nanotechnol. 6, 418-422 (2011).

[3] T. P. Almeida, et al., J. Nanopart. Res. 16:2395 (2014).

[4] J. M. De Teresa, et al., J. Phys. D: Appl. Phys. 49, 243003 (2016).

[5] A. Fernández-Pacheo, et al., Sci. Rep. 3, 1492 (2013).

[6] L. Serrano-Ramón, et al., ACS Nano., 5 (10), $7781-7787$ (2011).

[7] A. Fernández-Pacheo, et al., Appl. Phys. Lett. 94, 192509 (2009).

[8] R. Córdoba, et al. Microelectron. Eng. 87, 1550-1553 (2010).

[9] A. Fernández-Pacheo, et al., J. Phys. D: Appl. Phys. 42, 055005 (2009).

[10] T. P. Almeida, et al., Sci. Adv., 2, e1501801 (2016).

[11] T. P. Almeida, et al., Geophys. Res. Lett., 43, 8426-8434 (2016).

[12] L. Bernau et al., Angew. Chem. Int. Ed. 49, 8880-8884 (2010).

[13] R. I. Anishchenko, Phys. Stat. Sol. 18, 923 (1966).

[14] A. Sanchez and M. A. Ochando J. Phys. C: Solid State Phys. 18, 33 (1985).

[15] R. E. Dunin-Borkowski, et al., Ultramicroscopy, 74, 61-73 (1998).

[16] M. J. Hÿtch, et al., Phys. Rev. Lett., 91, 257207 (2003).

[17] R. K. K. Chong, et al., Inst. Phys. Conf. Ser., 179, 451-454 (2003).

[18] R. Córdoba, et al. Nanoscale Res. Lett., 6, 592 (2011).

[19] J. Pablo-Navarro, et al. ACS Appl. Nano Mater., 1, 38-46 (2018).

[20] L. Nagy, et al. PNAS, 114, 10356-10360 (2017).

\section{Acknowledgements}

The authors thank the University of Glasgow (200540-01) and Carnegie Trust (RIG007390) for funding. 